\title{
ON A COEFFICIENT INEQUALITY FOR STAR'LIKE FUNCTIONS
}

\author{
ROGER W. BARNARD
}

ABSTRACT. M. S. Robertson considered a coefficient in equality $I$ for starlike functions that, if true, would imply a generalized Bieberbach coefficient inequality $B$ for close to convex functions. An example is given of a starlike function whose coefficients do not satisfy coefficient inequality $l$.

Let $S$ denote the collection of regular and univalent functions $f(z)=$ $\sum_{n=1}^{\infty} A_{n} z^{n}$ in the unit disk $U$. Let $S^{*}$ denote the subclass of $S$ of functions $f$ such that $f(U)$ is starlike with respect to the origin. Let $K$ denote the close to convex functions in $S$, i.e. those functions $f \in S$ such that there exists a $g \in S^{*}$ and a real a such that $e^{i \alpha} z f^{\prime}(z) / g(z)=p(z)$, where $p(z)$ is a regular function of positive real part in $U$.

In [2] and [3] M. S. Robertson considered a generalized Bieberbach inequa lity:

$$
|n| A_{n}|-m| A_{m}|| \leq\left|n^{2}-m^{2}\right|\left|A_{1}\right|
$$

for $m$ and $n$ nonnegative integers. In [2] Robertson showed that (1) holds for all functions convex in one direction (for definition, see [2]), and that (1) holds for all functions in $K$ if $n-m$ is an even integer. In [1] J. A. Jenkins showed that the sharp upper bound for $3\left|A_{3}\right|-2\left|A_{2}\right|$ is $(5.02 \cdots)\left|A_{1}\right|$ when $f \in S$. It is still an open question whether (1) is true for all close to convex functions if $n-m$ is not an even integer. Robertson stated in [3] that (1) would hold for all functions in $K$ if every starlike function $f(z)=$ $z+\sum_{n=2}^{\infty} b_{n} z^{n},|z|<1$, has coefficients which satisfy

$$
\left|2 b_{n}-b_{2} b_{n-1}\right| \leq 2, \quad n=1,2,3, \cdots \quad\left(b_{0}=0\right) .
$$

In this note we give an example of a function in $S^{*}$ where (2) does not hold. Indeed, consider the function

Received by the editors January 7, 1974.

AMS (MOS) subject clas sifications (1970). Primary 30A34, 30A32.

Key words and phrases. Univalent functions, starlike functions, close to convex functions, coefficient inequalities. 


$$
F_{a}(z)=\left(\frac{1-z}{1+z}\right)^{a} \frac{z}{(1-z)^{2}}=z+\sum_{n=2}^{\infty} b_{n}(\alpha) z^{n}, \quad 0 \leq \alpha \leq 2
$$

where $b_{2}(\alpha)=2(1-\alpha), b_{3}(\alpha)=3-4 \alpha+2 \alpha^{2}, b_{4}(\alpha)=4\left(3-5 \alpha+3 a^{2}-\alpha^{3}\right) / 3$, and $b_{5}(\alpha)=\left(15-28 \alpha+22 \alpha^{2}-8 \alpha^{3}+2 \alpha^{4}\right) / 3$. Since $F_{0}(z)$ and $F_{2}(z)$ map $U$ univalently onto the complement of a radial ray, and $F_{a}(z), 0<\alpha<2$, maps $U$ univalently onto the complement of two radial rays, $F_{a} \in S^{*}$ for each $\alpha$, $0 \leq \alpha \leq 2$. If we let $T_{n}(\alpha)=2 b_{n}(\alpha)-b_{2}(\alpha) b_{n-1}(\alpha)$, then $T_{4}(0)=T_{5}(0)=$ $T_{5}(1)=T_{5}(2)=2$. It follows from the continuity of $\partial T_{4}(\alpha) / \partial \alpha$ that, since $\partial T_{4}(\alpha) /\left.\partial \alpha\right|_{a=0}>0,(2)$ does not hold for $F_{\alpha}(z)$ for some $\alpha>0$. In fact for $\alpha \neq 1$ and $0<a<2$, we have

$$
2<T_{5}(\alpha)=2+4 \alpha(2-\alpha)(1-\alpha)^{2} / 3 \leq T_{5}(1-\sqrt{2 / 2})=7 / 3 \text {. }
$$

It is of interest to note that if one considers the function $F \in K$ defined by $z F^{\prime}(z)=F_{a}(z) P(z)$, where $P(z)=(1+z) /(1-z)$, as might be suggested by the method of proof used by Roberts on in [2], that (1) holds for this function $F$.

\section{BIBLIOGRAPHY}

1. J. A. Jenkins, On an inequality considered by Robertson, Proc. Amer. Math. Soc. 19 (1968), 549-550. MR 37 \#01.

2. M. S. Robertson, A generalization of the Bieberbach coefficient problem for univalent functions, Michigan Math. J. 13 (1966), 185-192. MR 33 \#269.

3. - Quasi-subordination and coefficient conjectures, Bull. Amer. Math. Soc. 76 (1970), 1-9. MR $40 \# 4441$.

DEPARTMENT OF MATHEMATICS, TEXAS TECH UNIVERSITY, LUBBOCK, TEXAS 79409 